\title{
Perspectives on interferon-induced depression: toward an integration of psychiatry, neuroscience and immunology Peter Hauser
}

\author{
Address: Departments of Behavioral Neuroscience and Internal Medicine, Oregon Health \& Science University, Portland, Oregon, USA \\ from International Society on Brain and Behaviour: 3rd International Congress on Brain and Behaviour \\ Thessaloniki, Greece. 28 November - 2 December 2007 \\ Published: 17 April 2008 \\ Annals of General Psychiatry 2008, 7(Suppl I):S37 doi:I0.II86/1744-859X-7-SI-S37
}

This abstract is available from: http://www.annals-general-psychiatry.com/content/7/SI/S37

(c) 2008 Hauser; licensee BioMed Central Ltd.

Cytokines are small protein molecules which play a key role in viral clearance. Interferon-alpha (IFN) is a proinflammatory cytokine that is used to treat hepatitis C. However, the use of IFN can result in the development of neuropsychiatric side effects and sickness behavior, such as depression, irritability and fatigue. IFN-induced depression occurs in approximately $20-30 \%$ of patients with $\mathrm{HCV}$, yet the mechanisms and risk factors associated with this complication of its use are not well understood.

In patients chronically infected with the hepatitis $\mathrm{C}$ virus (HCV) and/or the human immunodeficiency virus (HIV) the there is a high prevalence of substance use and psychiatric disorders. Although, IFN-based therapies are the current treatment for patients with HCV, many patients with psychiatric or substance use disorders do not receive these therapies. Health care providers can be reluctant to treat these patients because of non-compliance concerns and neuropsychiatric side effects of IFN therapy, particularly depression. Increasingly, basic scientists, hepatologists, mental health and other health care providers have recognized the need for improved treatment strategies for HCV, one that addresses the co-entwined epidemics of chronic infection, substance abuse and psychiatric illness.

In general, IFN-induced depression worsens with increasing dose and duration of therapy. However, there are no established premorbid risk factors (e.g., the patient's past history, pre-therapy physical or psychiatric condition) that reliably predict the development of IFN-induced depression. Clinical experience and neurochemical and genetic research suggest underlying causes for the neuropsychiatric side effects induced by IFN and also suggest approaches to ameliorating those side effects. Antiviral therapy for patients with HCV is best provided through integrating medical, psychiatric, and substance abuse care.
Establishing care pathways, group education, and standardized screenings greatly enhances this approach.

Although there are gaps in our understanding of the optimal HCV treatment in patients with comorbid psychiatric and substance use disorders, in the future we must continue to expand our definition of HCV care to include these patients so that new management strategies and novel interventions can be developed and thus improve treatment outcomes for this underserved patient population. 